\title{
NAA10 variant in 38-week-gestation male patient: a case study
}

\author{
Antara Afrin, ${ }^{1}$ Jeremy W. Prokop, ${ }^{1,2}$ Adam Underwood, ${ }^{2,3}$ Katie L. Uhl, ${ }^{1,2}$ \\ Elizabeth A. VanSickle, ${ }^{4}$ Roja Baruwal, ${ }^{3}$ Morgan Wajda, ${ }^{3}$ Surender Rajasekaran, ${ }^{2,4}$ \\ and Caleb Bupp ${ }^{2,4}$ \\ ${ }^{1}$ College of Human Medicine, ${ }^{2}$ Department of Pediatrics and Human Development, Michigan State University, \\ Grand Rapids, Michigan 49503, USA; ${ }^{3}$ Walsh University, North Canton, Ohio 44720, USA; ${ }^{4}$ Medical Genetics, \\ Spectrum Health and Helen DeVos Children's Hospital, Grand Rapids, Michigan 49503, USA
}

Corresponding author: caleb.bupp@spectrumhealth.org

(C) 2020 Afrin et al. This article is distributed under the terms of the Creative Commons Attribution-NonCommercial

License, which permits reuse and redistribution, except for commercial purposes, provided that the original author and source are credited.

Ontology terms: biventricular hypertrophy; failure to thrive in infancy; hepatomegaly; intellectual disability; mild; progeroid facial appearance; sinus tachycardia; mesomelic/ rhizomelic limb shortening

Published by Cold Spring Harbor Laboratory Press

doi:10.1101/mcs.a005868
Abstract We present a male patient born at 38-wk gestation with rhizomelic shortening of extremities, hepatomegaly, ventriculomegaly, heart failure, severely depressed left ventricular function, biventricular hypertrophy, and biatrial enlargement. Additional physical findings included anteriorly displaced anus, vertebral anomalies, and brachydactyly. The patient's cardiac malformations led to persistent hypotension, sinus tachycardia, and multiorgan failure in the absence of arrhythmias. Rapid whole-exome sequencing was ordered on day of life (DOL) 8. The patient's family elected to withdraw supportive care, and he passed away that evening. Whole-exome sequencing returned posthumously and identified a variant in NAA10, E100K. The genotype-phenotype was closest to Ogden syndrome or amino-terminal acetyltransferase deficiency. Typical features of this rare X-linked syndrome include progeroid appearance, failure to thrive, developmental delays, hypotonia, and cardiac arrhythmias. Other family members were tested and the patient's mother, who has a history of mild intellectual disability, as well as a daughter born later, were identified as carriers. All carriers showed no cardiac findings. The carrier sister has manifested developmental delay and cortical atrophy. Protein modeling, evolution, dynamics, population variant assessments, and immunoprecipitation depict the deleterious nature of the variant on the interactions of NAA10 with NAA15. These findings had subsequent implications for posthumous diagnosis of the index patient, for female carriers, and regarding family planning. We highlight how these rapid genetic tests and variant characterization can potentially lead to informed decision-making between health-care providers and family members of patients with critical or lethal conditions when treatment options are limited.

[Supplemental material is available for this article.]

\section{INTRODUCTION}

The amino-terminal acetyltransferase A (NatA) complex is constituted of NAA10, a catalytic subunit, and NAA15, which docks NAA10 to the ribosome. The NatA complex acetylates the amino terminus of peptides as chains exit the ribosome in $40 \%$ of the human proteome (McTiernan et al. 2018). Variants of NAA10 can present as different rare genetic disorders. De novo variants can cause nonsyndromic developmental delay; however, others can present as syndromic developmental delay, such as Lenz microphthalmia syndrome and Ogden syndrome (Casey et al. 2015).

Ogden syndrome variants have been shown to have reduced catalytic activity on the NatA complex. In this X-linked syndrome, carrier mothers can be protected from phenotypic expressivity due to lyonization of the mutant-carrying chromosome ( $\mathrm{Wu}$ and Lyon 2018). 
Patients with NAA10-related syndrome can present with developmental, intellectual, and postnatal growth concerns that can be accompanied with cardiac pathology and skeletal anomalies. Males are more seriously affected, but female carriers may be asymptomatic or mildly to moderately impacted, likely related to X-inactivation (Rope et al. 2011).

In neonatal lethal syndromes, characterizing the phenotype within the limited lifespan is often challenging. Genetic testing results are often only available after the patient is deceased. This case illustrates the ability to make a rapid diagnosis of a rare sex-linked syndrome with future reproductive need for variant characterization, with patient phenotypes variable from those previously described.

\section{RESULTS}

In this case, the patient was born at 38-wk gestation via cesarean section. Prenatal ultrasound done for growth concerns at 31-wk gestation indicated bilateral ventriculomegaly. Anatomy ultrasound at 20-wk gestation had not identified any abnormalities. Fetal echocardiogram demonstrated cardiomegaly, right ventricular hypertrophy, and polyhydramnios. Primary HSV testing done during first trimester was $\mathrm{lgM} / \mathrm{lgG}$ negative. The patient was born with dysmorphic features, including low-set ears, retrognathia, proptosis, wide mouth, and rhizomelic shortening of arms and legs (Fig. 1A). The patient presented with hypertrichosis, hepatomegaly, sacral dimple, and wide-spaced toes. The anus was anteriorly displaced.

Laboratory results indicated persistent thrombocytopenia. Chest and abdominal X-rays showed spine and vertebral anomalies including minimal cervical vertebral body ossification, 13 thoracic vertebral bodies but 12 paired ribs, small upper thoracic vertebral bodies with asymmetry at T7/T8, and no ribs present at the eighth vertebral body. There was mild thoracic dextrocurvature at $\mathrm{T} 8$ and possible sagittal thoracic vertebral clefts (Fig. 1B). Newborn screen was normal. The patient demonstrated persistent mild lactic acidosis (2-3 mmol/L), low albumin, and hyperbilirubinemia (direct). Other tests were ordered to check for plasma amino acids, urine organic acids, plasma acylcarnitine profile, peroxisomal profile, and carbohydrate-deficient transferrin.
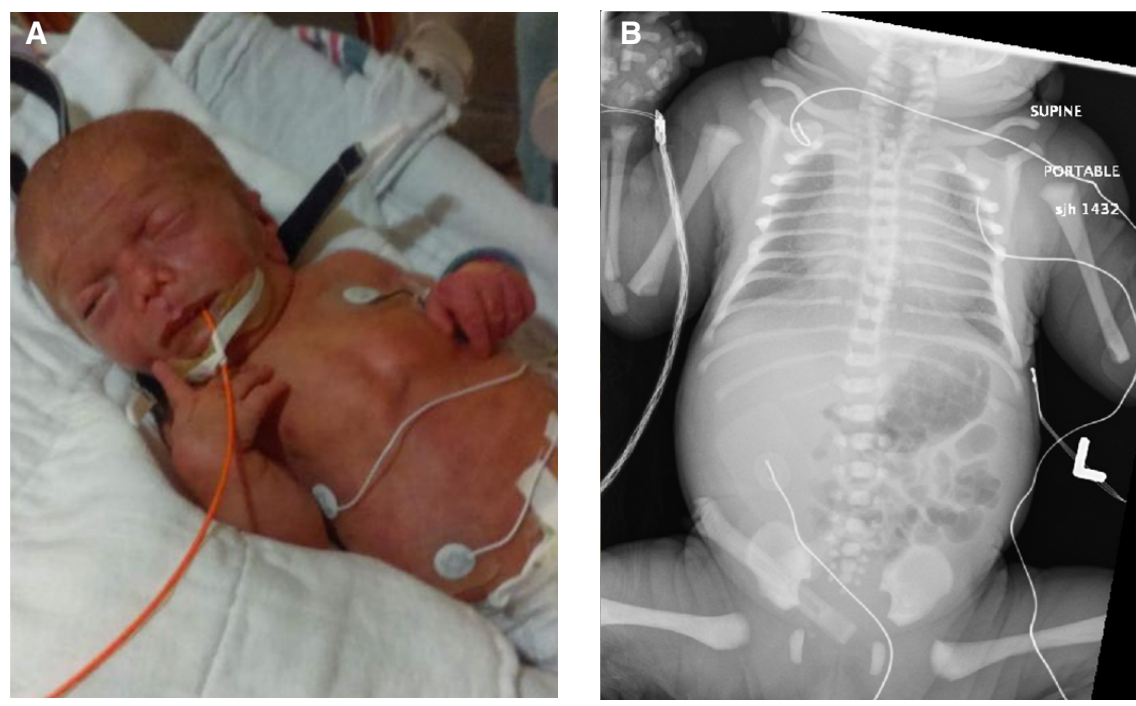

Figure 1. Clinical imaging. (A) Patient photograph from hospitalization showing aged appearance, low-set ears, retrognathia, proptosis, wide mouth, and some illustration of shortened upper limbs. (B) Chest X-ray image obtained shortly after birth showing skeletal anomalies of the spine, vertebrae, and ribs. 


\begin{tabular}{|c|c|c|c|c|c|c|c|c|}
\hline Gene & Chromosome & $\begin{array}{l}\text { HGVS DNA } \\
\text { reference }\end{array}$ & $\begin{array}{l}\text { HGVS protein } \\
\text { reference }\end{array}$ & $\begin{array}{l}\text { Variant } \\
\text { type }\end{array}$ & $\begin{array}{c}\text { Predicted } \\
\text { effect }\end{array}$ & Genotype & ClinVar ID & $\begin{array}{c}\text { Parent of } \\
\text { origin }\end{array}$ \\
\hline NAA10 & $x$ & $\begin{array}{l}\text { c. } 298 \mathrm{G}>\mathrm{A}, \\
\quad \mathrm{NM} \_003491.3\end{array}$ & p. E100K & Missense & Substitution & Hemizygous & VCV000986784.1 & Maternal \\
\hline
\end{tabular}

Urine glycosaminoglycan/oligosaccharides/mucopolysaccharides, lysosomal enzyme panel, and add-on $\beta$-glucuronidase enzyme for Sly syndrome studies were also conducted. The results of these tests were all normal. The patient developed cardiomyopathy and required ventilatory support. The poor cardiac function lead to hypotension and poor renal function, which resulted in acute kidney injury. Additionally, there were notable elevations in BUN and creatinine. These results led to a discussion about initiating ECMO support with the family.

The patient's older sister did not have similar presentation at birth and is normally developing. The patient's mother has a history of mild cognitive impairment and a history of global developmental delay as a child. She completed high school but required some assistance in school. No IQ testing was conducted. The patient's father does not have a significant medical history. Family history is otherwise noncontributory. Chromosome microarray of the patient was first obtained and was normal. Rapid whole-exome sequencing was then ordered on day of life (DOL) 8. The patient passed away later that evening and testing was switched to standard turnaround time. Whole-exome sequencing revealed a NAA10 variant that was labeled a variant of uncertain significance (VUS) (Table 1).

Family testing was pursued to identify female carriers and then evaluate their cardiac structure and function. Subsequent targeted testing of the family revealed that the patient's maternal grandmother was not a carrier of NAA10 E100K, but the patient's mother was a carrier of this variant. The patient's older sister did not have the NAA10 variant; however, gene sequencing of the sister born after the patient's death revealed that she was also a carrier of the NAA10 variant. This sibling does not have any cardiac findings to date (Fig. 2). She has subsequently manifested developmental delay (at $12 \mathrm{mo}$, no sitting, no pulling to stand, no words), thinning of the corpus callosum, mild prominence of lateral and third ventricles, and cortical atrophy. By familial report, these developmental findings are similar to her mother as a child. No brain imaging has been performed on the carrier mother. She developed oropharyngeal dysphagia with chronic cough that was causing failure to thrive affecting head circumference. A gastrostomy-jejunostomy tube was recently placed to help with feeding.

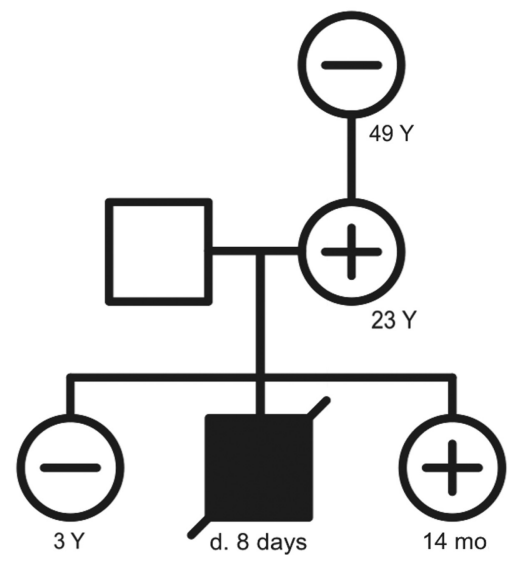

Figure 2. Family pedigree of NAA10 E100K. 
COLD SPRING HARBOR Molecular Case Studies
NAA10 variant in 38-wk gestation male patient
Review of historical records showed that the mother was diagnosed with moderate dysphagia at 18 mo of age with loss of growth velocity and smaller head circumference.

Once the patient passed away, the exome sequencing was changed from rapid analysis to standard turnaround time. The results returned $21 \mathrm{~d}$ after testing was initiated. The variant identified matched the clinical phenotype despite being classified as variants of uncertain significance. This merited an immediate discussion with the family as there were reproductive implications with the patient's mother being a carrier of the variant gene. Even though the family was dealing with grief at the recent loss of a child they did find comfort in the potential explanation for their loss. Inevitably, questions surrounding implications for their other daughter and future children arose. Their future daughter would be born almost $1 \mathrm{yr}$ later. Further analysis of this NAA10 variant is useful for this family to both aid the mourning process and provide future guidance.

Merging protein structures of NAA10 interacting with NAA15 with evolutionary analysis (Prokop et al. 2017) of open reading frame sequences in 139 species of NAA10 and 175 species of NAA15, we identified that E100 is found in a highly conserved pocket critical for the interaction (Fig. 3A). The interaction pocket consists of 199, E100, and N103 of NAA10 interacting electrostatically with K223 and R253 of NAA15 (Fig. 3B), all of which have a high conservation throughout evolution (Fig. 3C).

A total of 20 nsec of molecular dynamics simulations (MDS) was performed on both wildtype (WT) and E100K structures, showing equilibrium of the molecular movement (Fig. 4D). Analysis of the movements of atoms from residues around E100 showed that the E100K variant results in elevated molecular movement of the amino acid (Fig. 3E), suggesting loss of interaction with K223 and R253.

Further, we performed analysis of E100K predicted impact relative to all additional variants from ClinVar and gnomAD (Fig. 3F) integrating scores from PolyPhen-2, Provean, SIFT, Align-GVGD, and our conservation scoring system. The E100K ranks among the highest scoring, higher than many other ClinVar-annotated VUS and at similar values to the known NAA10 pathogenic and likely pathogenic annotated variants from ClinVar (Fig. 3F).

Therefore, we elected to perform biochemical assays on the impact of NAA10 E100K on the interaction with NAA15. Production and sequence confirmation of NAA10 and NAA10 E100K FLAG-tagged expression constructs was performed in $1 \mathrm{wk}$ using custom plasmid synthesis (DNA Atum). Overexpression of FLAG-tagged NAA10 WT or NAA10 E100K produce protein at even concentrations (Fig. 3G). These proteins were overexpressed with myc-tagged NAA15 and bound to FLAG-magnetic beads for coimmunoprecipitation (CoIP), determining that the E100K results in altered binding to NAA15 (Fig. $3 \mathrm{H}, \mathrm{I})$. Of interesting note is that the NAA10 E100K variant presents similar to S37P, seen in Ogden syndrome (Myklebust et al. 2014), in regard to location interacting with NAA15 (Fig. 4).

We then developed a three-dimensional printed protein model to show the physical relationship of NAA10 to NAA15 with the E100 site marked in red as an educational tool for the clinicians and the family (Fig. 5). The model can be ordered online as small and large prints at cost of production (https://www.shapeways.com/product/MLJE C3CLP/naa10?optionld=143806042\&li=shops). This model serves as an opportunity to educate additional families the pathogenicity behind Ogden Syndrome and the NAA10 protein interactions.

\section{DISCUSSION}

Rare disorders may have levels of rarity. Simply identifying a rare disorder can be an endeavor of happenstance and sometimes luck. Defining the spectrum of effect happens over time as more cases are identified and reported. The increasing availability of next-generation 


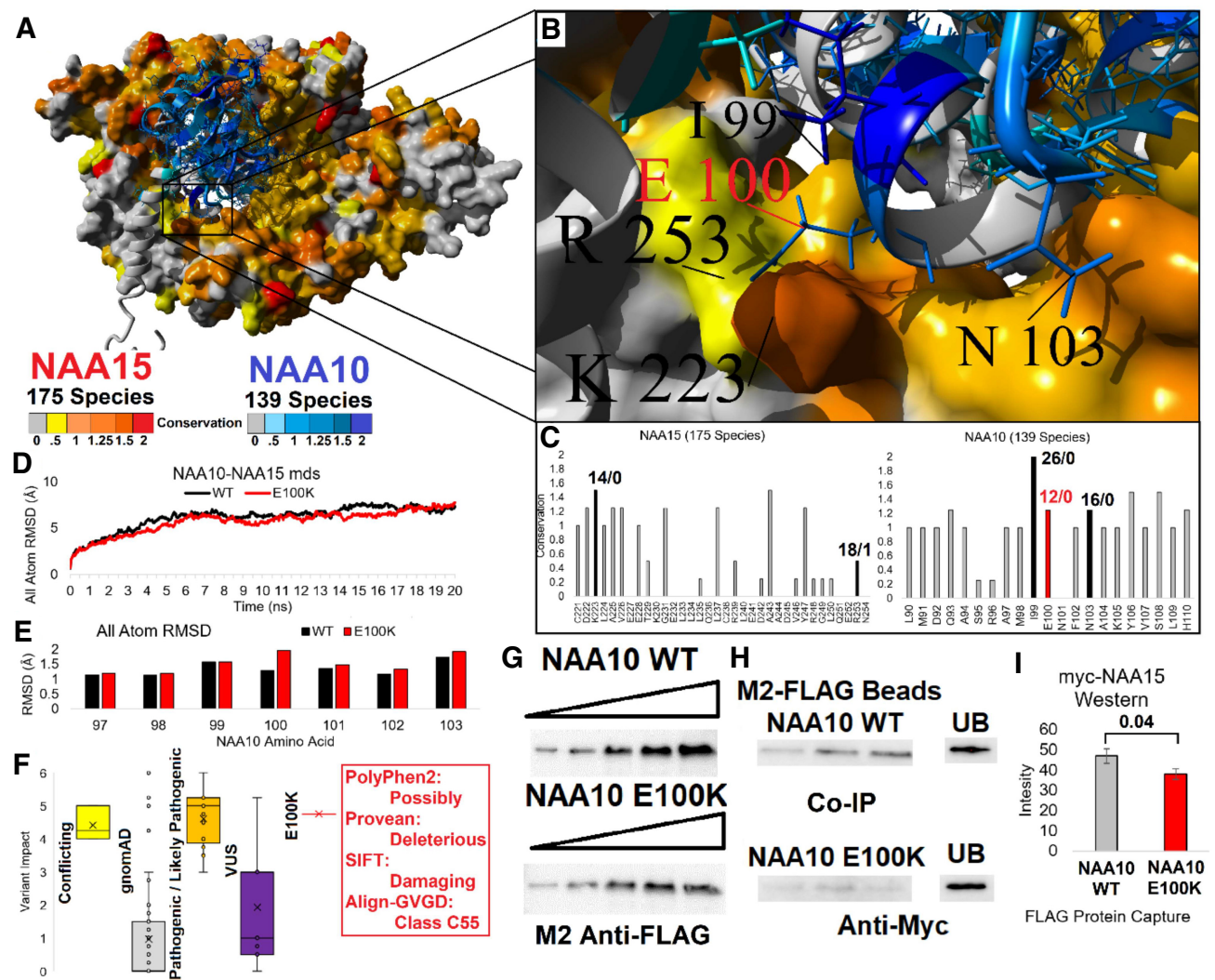

Figure 3. Characterization of NAA10 E100K. (A) Model of NAA10 (blue scale) interacting with NAA15 (red scale, surface plot). Color of amino acids are based on conservation scale of 0 (no conservation and gray) to 2 (top conserved and selected amino acids) of the indicated number of species. (B) Zoom-in view of NAA10 E100 site of contact with NAA15. Colors are same as A. (C) Conservation scores for NAA15 (left) and NAA10 (right) amino acids involved in contact. Numbers for each site are the synonymous/nonsynonymous changes throughout the evolutionary analysis. (D) Root mean squared deviation (RMSD) for all atoms averaged at each time point of a 20-nsec molecular dynamics simulation (mds) for either the wild-type (WT) or E100K model of NAA10 interacting with NAA15. (E) Average movement (RMSD) of each amino acids' atoms throughout the mds in either the WT or E100K of D. (F) Variant impact scores for all ClinVar variants (conflicting interpretation, yellow; pathogenic/likely pathogenic, orange; VUS, magenta), gnomAD (gray), and the E100K of patient (red). Different tools annotations are shown for E100K. (G) Overexpressed FLAG tagged NAA10 or NAA10 E100K proteins in cell culture with increasing levels of loaded protein. Western blots were performed using anti-FLAG M2 antibody (Sigma-Aldrich). (H) Coimmunoprecipitation (Co-IP) was performed with either NAA10WT or NAA10 E100K bound to M2 coated magnetic beads followed by washing, eluting, and western blot for the overexpressed myc-NAA15 using anti-myc antibody. (I) Quantification of six independent binding assays of NAA15 to NAA10 WT (gray) or NAA10 E100K (red).

sequencing and broad genomic testing is a major contributor, and that comes with the risk of variants of uncertain significance. Utilization of this testing in the critically ill neonatal population adds another layer with neonatal lethal conditions that previously would not have been diagnosed. Translating those VUSs to clinical action is a new challenge, particularly when significant pending morbidity and mortality enter the scenario. Outside of that scenario, familial grief is a posthumous symptom, which a genetic diagnosis may aid in treating. That scenario does not escape the impact of VUSs as this case illustrates.

The phenotype related to NAA10 continues to expand and gain clarity. Male patients remain more severely affected, although not as often this severe. The impact on females 
COLD SPRING HARBOR Molecular Case Studies

NAA10 variant in 38-wk gestation male patient

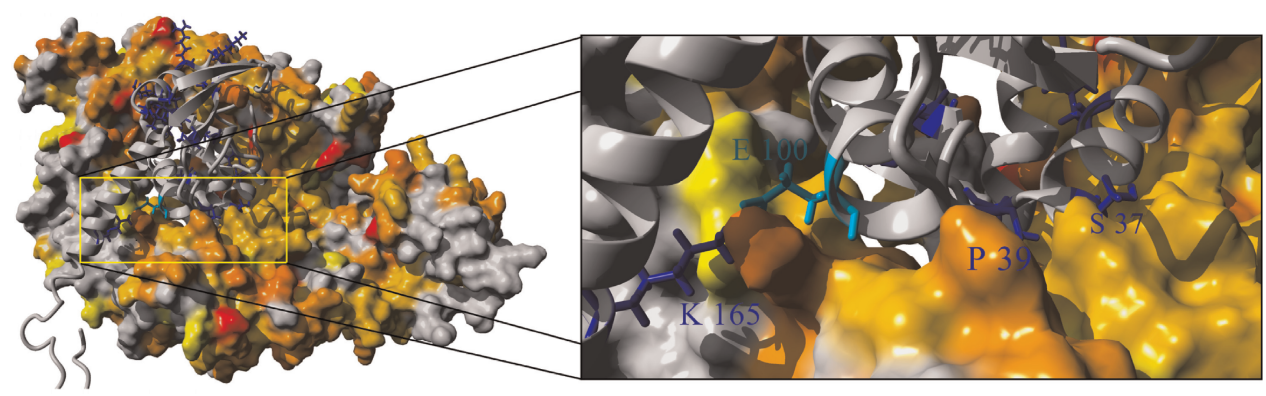

Figure 4. NAA10 pathogenic variants. NAA10 (gray) interacting with NAA15 (yellow to red color scale of Fig. 2A) showing the known ClinVar pathogenic variants (blue) or E100 of patient (cyan). The right side shows a zoom-in view of NAA15 contacts such as S37 and E100 (patient).

continues to be determined, with X-chromosome inactivation (XCl) an important factor. $\mathrm{XCl}$ also can differ in different tissue types. Clinically, testing patients for $\mathrm{XCl}$ can be challenging because of the paucity of clinical testing labs that perform this testing (Genetic Testing Registry lists five in United States and two internationally) and health insurance often denies this testing as not impacting treatment or management. In clinical practice, passing along this cost to patients is often prohibitive, which makes providing precise clinical care more challenging.

The patient's younger sister and mother's presentations reflect clinical phenotypes reported in 33 females who presented with either de novo missense variant or inherited variant in a familial case due to germline mosaicism. All 33 females had moderate to severe intellectual disability or global developmental delay with speech deficiencies. Hypotonia was also noted in the majority of the 33 female carriers of NAA10 variants. Although nonspecific, brain imaging revealed variable anomalies in these patients. Other anomalies included cardiac defects, facial dysmorphism, postnatal growth failure, and difficulties feeding (Saunier et al. 2016; Cheng et al. 2020).

Reclassification of variants is understandably a challenging process to find consensus and to standardize (Richards et al. 2015). Correlating test findings to phenotype can provide opportunity to see connections for patients, families, and providers when those may not truly exist. Therefore, the need to abide by agreed upon standards is important. For NAA10, recent publications have shown examples of how additional studies can be done with

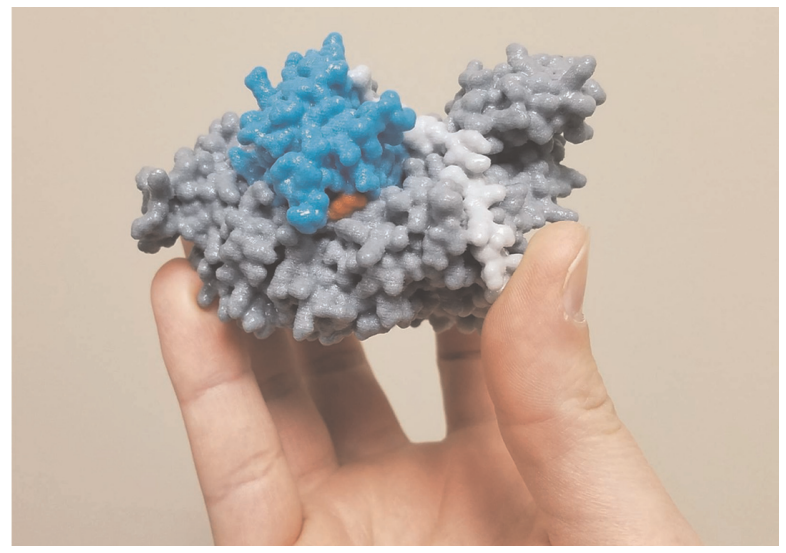

Figure 5. 3D-printed model of the variant. 
COLD SPRING HARBOR Molecular Case Studies
NAA10 variant in 38-wk gestation male patient enzymatic analysis to more thoroughly confirm a diagnosis and its phenotype, particularly when dealing with XCl (Cheng et al. 2020).

However, this case illustrates the limitations that can combine to leave patients, families, and providers at an impasse. Using computational methods and more broadly available and easy to utilize assays, VUS can be approached in a more efficient manner.

The posthumous results of this particular case went on to have subsequent familial implications. Moreover, the reproductive decisions being made from these genetics warrants variant reclassification of VUs, a vast need in clinical genetics, such that families can move forward with genomic diagnoses. It also informs future care for carrier females in this family. This family is planning to participate in further NAA10-specific research to help more fully understand this condition. It also bears mentioning the quandary that would have resulted if the results in this case had returned before the patient passed away that would have necessitated inclusion in a discussion of withdrawal of care.

Our pipeline of reclassification tools allows more thorough and nearer to real-time analysis of uncertain results to aid in the pathogenic determination. All of these allow providers and families to have more information for deciding upon treatment options and decisionmaking. This includes allowing families to withdraw care having a confirmed diagnosis with some understanding of the prognosis, thereby helping with that complex and deeply personal decision.

\section{METHODS}

\section{Whole-Exome Sequencing}

Whole blood was collected for whole-exome sequencing (WES) and sent to GeneDx (Supplemental Fig. 1).

\section{Protein Modeling and Evolution Assessment}

Protein modeling and evolution was performed as previous laid out by our laboratory (Prokop et al. 2017). In short, the PDB file 6C95 was parsed into NAA10 or NAA15 followed by homology modeling of the human sequence on each model. The models were complexed back together using 6C95 followed by energy minimization in YASARA using the YASARA2 force field (Krieger et al. 2002). Amino acid 100 was swapped for $K$ in YASARA and each (WT or E100K) structure saved as a PDB file. The PDB files were run for 20 nsec of molecular dynamics simulations in YASARA using the AMBER14 force field, $\mathrm{pH}=7.4$, $\mathrm{NaCl}$ at 0.9 mass fraction, water density at $0.997 \mathrm{~g} / \mathrm{mL}$, periodic boundaries, and long-range coulomb forces.

Evolutionary analysis of NAA10 or NAA15 was performed by extracting the sequences from NCBI for all species known. Sequences were parsed for open reading frames using TransDecoder. Sequences were aligned using ClustalW codon followed by removing any sequences with ambiguity (ns) or gaps where $>90 \%$ of other species have sequences. The clean alignment was then condensed down to human sequence numbering, removing any gaps in the alignment found for human sequence. In MEGA (Tamura et al. 2011), codon selection was assessed using the Muse-Gaut model (Muse and Gaut 1994) of codon substitution and the Tamura-Nei model (Tamura and Nei 1993) of nucleotide substitution using the HyPhy software package (Pond et al. 2005).

\section{Genomic Profiling of NAA10 Variants}

Variants were extracted from gnomAD (Karczewski et al. 2019) (https://gnomad .broadinstitute.org/) or ClinVar (Landrum et al. 2016) (https://www.ncbi.nlm.nih.gov/ 
clinvar/) for NAA10 on 1/20/2020. Each missense or nonsense variant was run through PolyPhen-2 (Adzhubei et al. 2010), Provean (Choi and Chan 2015), SIFT (Choi and Chan 2015), and AlignGVGD (Mathe et al. 2006). Scores for damaging calls in each were given 1 and added for all the tools with our conservation score, for a max of 6. Clinical annotations from ClinVar were used to bin scores for Pathogenic/Likely pathogenic, VUSs, and conflicting interpretations and compared to those in gnomAD that were not present in ClinVar.

\section{Cloning}

Plasmids for mammalian NAA10 overexpression were custom synthesized with DNA2.0 into the pD663-Arc plasmid. The NAA10 open reading frame was codon optimized for human cells with an additional TEV cleavable sequence followed by a 3XFLAG tag. The construct was cloned in frame into the 2A-RFP site to allow for ribosomal separation of an RFP that can be used for transfection efficiency. The NAA10 E100K was synthesized the same as wild type through DNA2.0. The NAA15 expression plasmid was obtained from OriGene with a Myc-DDK-tag (OriGene: MR211001).

\section{Co-IP}

HeLa cells were seeded at $1 \times 10^{4}$ cells $/ \mathrm{cm}^{2}$ in six-well plates (TPP tissue culture plates) in DMEM (Sigma-Aldrich) supplemented with 10\% fetal bovine serum (Atlanta Biologicals) in a humidified chamber at $37^{\circ} \mathrm{C}$ and $5 \% \mathrm{CO}_{2}$. After $24 \mathrm{~h}, 1.5 \mu \mathrm{g}$ of total plasmid DNA was transiently cotransfected into each well using ViaFect Transfection Reagent (Promega) at a 4:1 reagent to DNA ratio in serum free medium according to reagent protocol. All NAA10 and NAA15 cotransfections were completed in triplicate. Each well was transfected with $750 \mathrm{ng}$ NAA10 construct (WT or E100K) and $750 \mathrm{ng}$ of NAA15. After $30 \mathrm{~h}$, cells were washed twice in $3 \mathrm{~mL}$ of $37^{\circ} \mathrm{C}$ modified Dulbecco's Phosphate Buffered Saline $(140 \mathrm{mM} \mathrm{NaCl}, 8 \mathrm{mM}$ $\mathrm{Na}_{2} \mathrm{HPO}_{4}, 2 \mathrm{mM} \mathrm{KH}{ }_{2} \mathrm{PO}_{4}, 10 \mathrm{mM} \mathrm{KCl}, \mathrm{pH}$ 7.3) and $300 \mu \mathrm{L}$ ice-cold Pierce IP Lysis Buffer with Halt Protease Inhibitor Cocktail added to a $2 \times$ final concentration (ThermoFisher) and incubated $20 \mathrm{~min}$ at $4^{\circ} \mathrm{C}$. Cell lysates were transferred to chilled $1.5-\mathrm{mL}$ microfuge tubes and centrifuged $14,000 \mathrm{~g}$ for $10 \mathrm{~min}$ at $4^{\circ} \mathrm{C}$ followed by a BCA protein assay (ThermoFisher).

Anti-FLAG M2 conjugated Dyna beads (Sigma-Aldrich), $25 \mu \mathrm{L}$ per native or variant bait NAA10 well, were washed twice in $250 \mu \mathrm{L}$ chilled modified PBS containing $2 \times$ Protease Inhibitor Cocktail. An amount of $2 \mathrm{mg}$ of cleared lysate containing the $3 \times$ FLAG-tagged bait proteins NAA10 or NAA10 E100K and the Myc-tagged NAA15 prey protein were incubated at $4^{\circ} \mathrm{C}$ on a rotator in a total volume of $400 \mu \mathrm{L}$ IP Buffer. After $1 \mathrm{~h}$, the unbound fraction of lysate was collected in a $1.5-\mathrm{mL}$ tube and beads were washed three times with $400 \mu \mathrm{L}$ fresh IP buffer containing $2 \times$ protease inhibitor. After collecting each wash, $60 \mu \mathrm{l}$ of $2 \times \mathrm{SDS}$ sample (4\% SDS, 20\% glycerol, 0.004\% bromophenol blue, 125 mM Tris-Cl, 150 mM DTT, 2× Protease Inhibitor Cocktail, pH 6.8) was added to the immunocomplexes and transferred to $200-\mu \mathrm{L}$ tubes. An amount of $100 \mu \mathrm{L}$ of NAA15 lysate was retained to serve as a positive control; $10 \mu \mathrm{L}$ from each unbound fraction, and wash was pooled for SDS-PAGE. An amount of $10 \mu \mathrm{L}$ of each pooled unbound fraction and the third wash was transferred to a $200-\mu \mathrm{L}$ tube with $10 \mu \mathrm{L}$ of $2 \times \mathrm{SDS}$ sample buffer. All samples were incubated for $12 \mathrm{~min}$ at $99^{\circ} \mathrm{C}$, and $20 \mu \mathrm{L}$ of each was loaded on an Any kD Mini-PROTEAN TGX Precast Gel (BIORAD). Proteins were electrophoresed for $25 \mathrm{~min}$ at $250 \mathrm{v}$ in chilled Tris-glycine-SDS running buffer and then transferred to PVDF membranes at $2 \mathrm{~mA} / \mathrm{cm}^{2}$ for $90 \mathrm{~min}$. Membranes were then blocked $45 \mathrm{~min}$ at $22^{\circ} \mathrm{C}$ in Tris buffered saline (50 mM Tris-Cl, $150 \mathrm{mM} \mathrm{NaCl}, \mathrm{pH}$ 7.5) containing $0.05 \%$ Tween20 (TBST) and 4\% nonfat dry milk. Overexpressed FLAG-tagged NAA10 and NAA10 E100K proteins were detected using an anti-FLAG M2 antibody (Sigma-Aldrich) diluted 1:4500 in blocking solution. Immunoreactive Myc-tagged NAA15 was detected with a rabbit antiMyc Tag HRP Conjugated Antibody (Bethyl A190-105P) diluted 1:20,000 in blocking 
Competing Interest Statement The authors have declared no competing interest.

Received October 1, 2020; accepted in revised form October 30, 2020. solution and incubated $30 \mathrm{~h}$ at $4^{\circ} \mathrm{C}$. After three 25 -min washes in TBST, blots were then rinsed three times in reverse osmosis (RO) water and then placed in a bag with $1300 \mu \mathrm{L}$ SuperSignal West Femto chemiluminescent substrate (ThermoFisher). Images were then collect on a ChemiDoc MP Imagining System (BIORAD). Quantification of each band was performed in ImageJ followed by assessing values with a T-test for significance.

\section{D Protein Models}

The WT NAA10-NAA15 PDB model from above was loaded into PyMOL. The NAA10 subunit was colored cyan and the NAA15 unit was colored gray. The surfaces were shown as solvent accessible for each. Amino acids 95-105 of NAA10 were colored red to identify the binding pocket. The structure was then exported as a VRML2 file in PyMOL and the VRML2 file was uploaded into ShapeWays followed by setting different sizing and selecting glossy full-color sandstone printing. These files are made available on the ProkopLab ShapeWays account (https://www.shapeways.com/shops/prokoplab) for non-marked-up printing to the public.

\section{ADDITIONAL INFORMATION}

\section{Data Deposition and Access}

Consent for data sharing for this patient could not be obtained and thus the data are not publicly available. The variant was submitted to ClinVar (https://www.ncbi.nlm.nih.gov/clinvar/) and can be found under accession number VCV000986784.1.

\section{Ethics Statement}

Patient consent for sequencing was obtained orally. The work was not required to be performed under an IRB.

\section{Acknowledgments}

The authors wish to thank the family for their participation in this study.

\section{Author Contributions}

S.R. and C.P.B. designed the study and developed the protocol. J.W.P., A.U., K.L.U., R.B., and M.W. performed variant interpretation. C.P.B. investigated the patient. A.A., E.A.V., and C.P.B. created the draft of the manuscript. All authors reviewed all drafts of the manuscript including the final draft.

\section{REFERENCES}

Adzhubei IA, Schmidt S, Peshkin L, Ramensky VE, Gerasimova A, Bork P, Kondrashov AS, Sunyaev SR. 2010. A method and server for predicting damaging missense mutations. Nat Methods 7: 248-249. doi:10.1038/ nmeth0410-248

Casey JP, Støve SI, McGorrian C, Galvin J, Blenski M, Dunne A, Ennis S, Brett F, King MD, Arnesen T, et al. 2015. NAA10 mutation causing a novel intellectual disability syndrome with long QT due to N-terminal acetyltransferase impairment. Sci Rep 5: 16022. doi:10.1038/srep16022

Cheng H, Gottlieb L, Marchi E, Kleyner R, Bhardwaj P, Rope AF, Rosenheck S, Moutton S, Philippe C, Eyaid W, et al. 2020. Phenotypic and biochemical analysis of an international cohort of individuals with variants in NAA10 and NAA15. Hum Mol Genet 29: 877-878. doi:10.1093/hmg/ddz173

Choi Y, Chan AP. 2015. PROVEAN web server: a tool to predict the functional effect of amino acid substitutions and indels. Bioinformatics 31: 2745-2747. doi:10.1093/bioinformatics/btv195 
Karczewski KJ, Francioli LC, Tiao G, Cummings BB, Alföldi J, Wang Q, Collins RL, Laricchia KM, Ganna A, Birnbaum DP, et al. 2019. Variation across 141,456 human exomes and genomes reveals the spectrum of loss-of-function intolerance across human protein-coding genes. BioRxiv 531210. doi:10.1101/531210

Krieger E, Koraimann G, Vriend G. 2002. Increasing the precision of comparative models with YASARA NOVAa self-parameterizing force field. Proteins 47: 393-402. doi:10.1002/prot.10104

Landrum MJ, Lee JM, Benson M, Brown G, Chao C, Chitipiralla S, Gu B, Hart J, Hoffman D, Hoover J, et al. 2016. ClinVar: public archive of interpretations of clinically relevant variants. Nucleic Acids Res 44: D862-D868. doi:10.1093/nar/gkv1222

Mathe E, Olivier M, Kato S, Ishioka C, Hainaut P, Tavtigian SV. 2006. Computational approaches for predicting the biological effect of p53 missense mutations: a comparison of three sequence analysis based methods. Nucleic Acids Res 34: 1317-1325. doi:10.1093/nar/gkj518

McTiernan N, Støve SI, Aukrust I, Mårli MT, Myklebust LM, Houge G, Arnesen T. 2018. NAA10 dysfunction with normal NatA-complex activity in a girl with non-syndromic ID and a de novo NAA10 p.(V111G) variant: a case report. BMC Med Genet 19: 47. doi:10.1186/s12881-018-0559-z

Muse SV, Gaut BS. 1994. A likelihood approach for comparing synonymous and nonsynonymous nucleotide substitution rates, with application to the chloroplast genome. Mol Biol Evol 11: 715-724.

Myklebust LM, Damme PV, Støve SI, Dörfel MJ, Abboud A, Klavik TV, Grauffel C, Jonckheere V, Wu Y, Swensen J, et al. 2014. Biochemical and cellular analysis of Ogden syndrome reveals downstream Nt-acetylation defects. Hum Mol Genet 7: 1956-1976. doi:10.1093/hmg/ddu611

Pond SLK, Frost SDW, Muse SV. 2005. HyPhy: hypothesis testing using phylogenies. Bioinformatics 21: 676 679. doi:10.1093/bioinformatics/bti079

Prokop JW, Lazar J, Crapitto G, Smith DC, Worthey EA, Jacob HJ. 2017. Molecular modeling in the age of clinical genomics, the enterprise of the next generation. J Mol Model 23: 75. doi:10.1007/s00894-0173258-3

Richards S, Aziz N, Bale S, Bick D, Das S, Gastier-Foster J, Grody WW, Hegde M, Lyon E, Spector E, et al. 2015. Standards and guidelines for the interpretation of sequence variants: a joint consensus recommendation of the American College of Medical Genetics and Genomics and the Association for Molecular Pathology. Genet Med 17: 405-423. doi:10.1038/gim.2015.30

Rope AF, Wang K, Evjenth R, Xing J, Johnston JJ, Swensen JJ, Johnson WE, Moore B, Huff CD, Bird LM, et al. 2011. Using VAAST to identify an X-linked disorder resulting in lethality in male infants due to $\mathrm{N}$-terminal acetyltransferase deficiency. Am J Hum Genet 89: 28-43. doi:10.1016/j.ajhg.2011.05.017

Saunier C, Støve SI, Popp B, Gérard B, Blenski M, Ahmew N, Bie CD, Goldenberg P, Isidor B, Keren B, et al. 2016. Expanding the phenotype associated with NAA10-related N-terminal acetylation deficiency. Hum Mutat 37: 755-764. doi:10.1002/humu.23001

Tamura K, Nei M. 1993. Estimation of the number of nucleotide substitutions in the control region of mitochondrial DNA in humans and chimpanzees. Mol Biol Evol 10: 512-526.

Tamura K, Peterson D, Peterson N, Stecher G, Nei M, Kumar S. 2011. MEGA5: molecular evolutionary genetics analysis using maximum likelihood, evolutionary distance, and maximum parsimony methods. Mol Biol Evol 28: 2731-2739. doi:10.1093/molbev/msr121

Wu Y, Lyon GJ. 2018. NAA10-related syndrome. Exp Mol Med 50: 85. doi:10.1038/s12276-018-0098-x 


\section{COLD SPRING HARBOR Molecular Case Studies}

\section{NAA10 variant in 38-week-gestation male patient: a case study}

Antara Afrin, Jeremy W. Prokop, Adam Underwood, et al.

Cold Spring Harb Mol Case Stud 2020, 6: a005868

Access the most recent version at doi: $10.1101 / \mathrm{mcs} . a 005868$ Supplementary http://molecularcasestudies.cshlp.org/content/suppl/2020/12/17/mcs.a005868.D
Material

References This article cites 19 articles, 1 of which can be accessed free at: http://molecularcasestudies.cshlp.org/content/6/6/a005868.full.html\#ref-list-1

License This article is distributed under the terms of the Creative Commons Attribution-NonCommercial License, which permits reuse and redistribution, except for commercial purposes, provided that the original author and source are credited.

Email Alerting Receive free email alerts when new articles cite this article - sign up in the box at the Service top right corner of the article or click here. 